\title{
Recent progress in understanding deconfinement and chiral restoration phase transitions
}

\author{
Edward Shuryak ${ }^{1, a}$ \\ ${ }^{1}$ Department of Physics and Astronomy, Stony Brook University Stony Brook NY 11794 USA
}

\begin{abstract}
Paradigme shift in gauge topology, from instantons to their constituents instanton-dyons - has recently lead to very significant advances. Like instantons, they have fermionic zero modes, and their collectivization at sufficiently high density explains the chiral symmetry breaking. Unlike instantons, these objects have electric and magnetic charges. Their back reaction on the mean value of the Polyakov line (holonomy) allows to explain the deconfinement transition. The talk summarizes recent works on the dyon ensemble, done in the mean field approximation (MFA), and also by direct numerical statistical simulation. Introduction of non-trivial quark periodicity conditions leads to drastic changes in both deconfinement and chiral transitions. In particulaly, in the so called $Z\left(N_{c}\right)-Q C D$ model the former gets much stronger, while the latter does not seem to occur at all.
\end{abstract}

\section{Introduction}

Confinement is the most famous non-perturbative feature of the gauge theories. Its most intuitive explanation from 1970's is the dual superconductor model by Nambu, 't Hooft and Mandelstam [1]. Lattice studies did reveal the monopoles (albight defined in certain gauges) and prove that they indeed form Bose-Einstein condensate at $T<T_{c}$, see e.g. [3].

Original discussion of the chiral symmetry and its breaking predate QCD and even quarks. In a classic paper Nambu and Jona-Lasinio [2] showed that an attraction in the scalar $\bar{q} q$ channel, if strong enough, can dynamically "gap" the surface of the Dirac sea. The origin of this interaction - claimed to be the origin of the mass of the "constinuent quarks", the nucleons and thus ourselves - was of course in 1961 completely unknown. Two decades later, in my paper [4], it was suggested that NJL attraction is nothing elase but 't Hooft effective $2 N_{f}$ interaction induced by instantons. Two parameters of the NJL model has been substituted by another two: the total instanton-antiinstanton density $n \sim 1 / \mathrm{fm}^{4}$ and their typical size $\rho \sim 1 / 3 \mathrm{fm}$. Of course, 't Hooft vertex does more than the NJL operator: in particular, it breaks the $U(1)_{A}$ symmetry explicitly.

A decade later instantons were found and studied on the lattice. Statistical mechanics of instanton ensemble, including 't Hooft interaction to all orders, known as the Interacting Instanton Liquid Model, has been developed and solved numerically in 1990's, for a review see [5]. Among other things, it introduced the notion of "collectivized zero mode zone", or ZMZ for short. Lattice practitioners struggle with it till now, since most of numerical fluctuations in simulations come from it.

\footnotetext{
a e-mail: Edward.Shuryak@stonybrook.edu
} 
And yet, some important questions remained unanswered, such as e.g. Is there any connection between confinement and chiral symmetry breaking? Why is it that the corresponding finite temperature transitions happen close, $T_{c} \approx T_{\chi}$ for fundamental quarks, but not for adjoint ones [29] ? Apart of color representations and the number of flavors, can one introduce other parameters, affecting these transitions and revealing the underlying mechanism?

The so called Polyakov line is used as a deconfinement order parameter, being nonzero at $T>T_{c}$. Interpreting this as existence of nonzero average $A_{0}$ field, one needs to modify all clasical solutions respectively. When such solutions were found in $1998[6,7]$ it has been realized that instantons get split into $N_{c}$ (number of colors) constituents, the selfdual instanton-dyons ${ }^{1}$, connected only by (invisible) Dirac strings. Since these objects have nonzero electric and magnetic charges and source Abelian (diagonal) massless gluons, the corresponding ensemble is an "instanton-dyon plasma", with long-range Coulomb-like forces between constituents.

The first application of the instanton-dyons were made soon after their discovery in the context of supersymmetric gluodynamics [10]. This paper solved a puzzling mismatch (by the factor 2) of the value of the gluino condensate, between the instanton-based and general supersymmetric evaluations of it.

Diakonov and collaborators (for review see [8] ) emphasized that, unlike the (topologically protected) instantons, the dyons interact directly with the holonomy field. They suggested that since such dyon (anti-dyon) become denser at low temperature, their back reaction may overcome perturbative holonomy potential and drive it to its confining value, leading to vanishing of the mean Polyakov line, or confinement. Specifically, Diakonov and collaborators focused on the self-dual sector $L, M$ and studied the one-loop contribution to the partition function [9]. The volume element of the moduli space was written in terms of dyons coordinates as a determinant of certain matrix $G$, to be referred to as Diakonov determinant. In a dilute limit it leads to Coulomb interactions between the dyons, but in the dense region it becomes strongly repulsive, till at certain density the moduli volume vanishes.

A semi-classical confining regime has been defined by Poppitz et al $[11,12]$ in a carefully devised setting of softly broken supersymmetric models. While the setting includes a compactification on a small circle, with weak coupling and an exponentially small density of dyons, the minimum at the confining holonomy value is induced by the repulsive interaction in the dyon-antidyon molecules (called bions by these authors). The crucial role of the supersymmetry is the cancellation of the perturbative Gross-Pisarski-Yaffe-Weiss (GPYW) [13] holonomy potential: as a result, in this setting there is no deconfined phase with trivial holonomy at all, unless supersymmetry is softly broken. Sulejmanpasic and myself [14] proposed a simple analytic model for the dyon ensemble with dyonantidyon "repulsive cores", and have shown how they may naturally induce confinement in dense enough dyonic ensemble.

Recent progress to be discussed below is related to studies of the instanton-dyon ensembles. We will focus on a series of papers devoted to high-density phase and mean field approximation [16-20] in section 3, and on the direct numerical simulation of the dyon ensembles [21-25] in section 4 .

Important ingredient of both of them is classical dyon-antidyon interaction, determined Larsen and myself in Ref[22]: its discussion we skip for space reasons. We would like to emphasize also importance of studies devoted to the instanton-dyon identification on the lattice, such as [26].

\footnotetext{
${ }^{1}$ They are called "instanton-monopoles" in applications to supersymmetric settings, e.g. by Khose et al and Unsal et al. Similar (but no idenical) objects were called the "instanton quarks" by Zhitnitsky et al.
} 


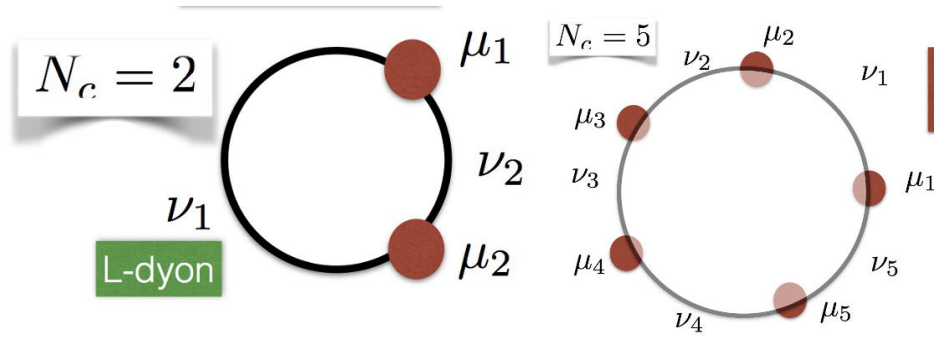

Figure 1. The holonomy circle and the definition of the parameters $\mu_{i}$ and $v_{i}$, for 2 and 5 colors.

\section{Non-zero holonomy, instanton-dyons and confinement}

The "holonomy" issue refers to the observation that a (gauge invariant) Polyakov loop

$$
P=P \exp \left(i \int_{0}^{\beta} A_{0}^{a} T^{a} d \tau\right)
$$

has a nonzero vacuum expectation value (VEV), $\langle P>\neq 0$. Note that there is no trace in this formula, so it is some unitary color matrix. One can always gauge rotate it to be a diagonal one, to Cartan sub-algebra with $N_{c}-1$ parameters. The corresponding phases are denoted $2 \pi \mu_{i}$, and their subsequent differences $v_{i}=\mu_{i+1}-\mu_{i}$. Fig. 1 explains these notations, for 2 and 5 colors. Note that $\sum \mu_{i}=0$ and $\sum v_{i}=1$ : thus $v_{i}$ are fractions of the holonomy circle. The action and the topological charge of the $N_{c}$ dyond is $v_{i}$ times that of the instanton.

For the simplest $S U(2)$ color group to be discussed below there is only one diagonal generator $T^{3}$ and only one parameter: The notations to be used below are $v=v_{2}, \bar{v}=1-v=v_{1}$. The mean Polyakov line is in this notation simply

$$
<\frac{1}{2} \operatorname{Tr} P>=\cos (\pi v)
$$

At high $T<P>\rightarrow 1$, which means all $\mu_{i} \rightarrow 0$. Thus all but one $v_{i} \rightarrow 0$, and one tends to 1: so the original instanton action is recovered. In the temperature interval (1..3) $T_{c}$ the mean Polyakov line or $v(T)$ is a smooth function of the temperature, changing from 0 to 1 . Accounting for this phenomenon lead Pisarski and collaborators to "semi-QGP" paradigm [15] and eventually led to the construction of the PNJL model, in which light quark paths are weighted by $<P\left(T>\right.$. At $T<T_{c}$, in a confined phase, $\langle P\rangle=0$ which means that $v=1 / 2$.

A nontrivial average value of the Polyakov line $\langle P\rangle \neq 1$, indicating that an expectation value of the gauge potential is nonzero. This calls for re-defining the boundary condition of $A_{4}$ at infinity, for any solitons made of gauge fields, including the instantons.

For the SU(2) gauge group the selfdual ones are called $M$ with charges $(e, m)=(+,+)$ and $L$ with charges $(e, m)=(-,-)$, the anti-selfdual antidyons are called $\bar{M},(e, m)=(+,-)$ and $\bar{L},(e, m)=(-,+)$.

\section{Dense dyon plasma in the mean field approximation}

The first paper of the series, by Liu, Zahed and myself [16], had established the mean field approximation (MFA) in the technical sense. The derivation is rather traditional: after bosonization of the partition function, certain fields are decleared to be $x$-independent parameters, over which the free 
energy is minimized. The derivatives over all parameters define the so called "gap equations", which needs to be solved together, defining their values at the global minimum.

Physics-wise, the main idea is that if the ensemble of the dyons is dense enough, strong electric screening appears, which effectively reduces the pair-wise correlations in the system, in favor of some average mean field. Here there is no place to present technical details of these works, and we just summarize the results.

It is shown, that dense enough dyon ensemble does shift the minimum of the holonomy potential to the confining value, $v=1 / 2$ for the SU(2) gauge theory considered.

The next work of the series [17] applies MFA to the $N_{c}=2$ color theory with $N_{f}=2$ light quark flavors. At high density the minimum of the free energy still corresponds to the confining ensemble with $v=1 / 2$. The gap equation for the effective quark mass (or the quark condensate) of [17] the usual form

$$
\int \frac{d^{3} p}{(2 \pi)^{3}} \frac{M^{2}(p)}{p^{2}+M^{2}(p)}=n_{L}
$$

where the r.h.s. is the $L$-dyon density. The equation is for the parameter $\lambda$ in the mass function $M(p)=$ $\lambda p T(p)$, in which $T(p)$ being the Fourier transform of the "hopping matrix element" calculated using the fermionic zero mode. Momentum dependence of $M(p) / \lambda$ is shown in Fig.2: note that its shape and the behavior at small momenta is different from that of an instanton. The solution for the condensate can be parameterized as

$$
\frac{|<\bar{q} q>|}{T^{3}} \approx 1.25\left(\frac{n_{L}}{T^{3}}\right)^{1.63}
$$

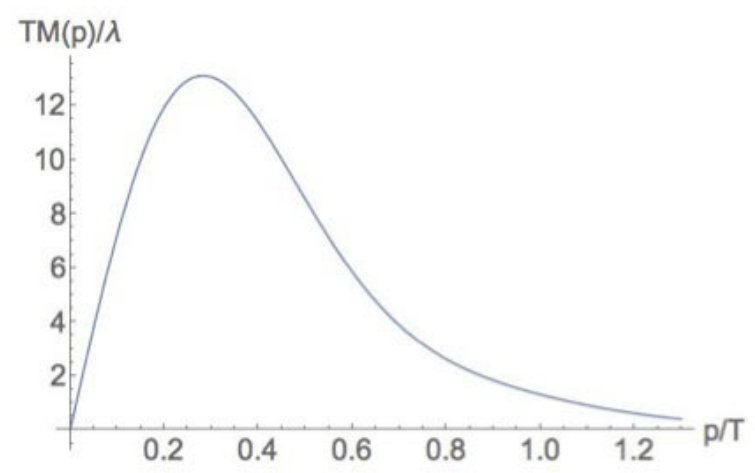

Figure 2. The momentum dependent constituent quark mass $T M(p) / \lambda$ versus momentum in units of temperature $p / T$.

A generalization of the mean field treatment to arbitrary number of colors and flavors in [17] shows that this gap equation has nonzero solutions for the quark condensate only if

$$
N_{f}<2 N_{c}
$$

So, the critical number of flavors is $N_{f}=6$ for $N_{c}=3$. The lattice simulation indeed show weakening of chiral symmetry violation effects with increasing $N_{f}$, but specific results about on the end of chiral symmetry breaking are so far rather incomplete: for $N_{c}=3$ we know that in the $N_{f}=4$ case the chiral 
symmetry is broken, the case $N_{f}=8$ is not yet completely decided and $N_{f}=12$ seems to be already in the conformal window.

Another important generalization - for quarks in the adjoint representation - is made in a separate paper [19]. The number of fermionic zero modes increases, and they are more comlicated. In the symmetric dense phase both $\mathrm{M}$ and $\mathrm{L}$ dyons have two zero modes. But the actual difficulty is not some longer expressions but the fact that one of them has rather singular behavior exactly at the confining value of the holonomy, $v=1 / 2$, so approaching it needs special care. In the case $N_{c}=2, N_{a}=1$ the deconfinement and chiral restoration happen at about the same temperature.

\section{Statistical simulations of the instanton-dyon ensembles}

The first direct simulation of the instanton-dyon ensemble with dynamical fermions has been made by Faccioli and myself in [21]. The general setting follows the example of the "instanton liquid", it included the determinant'of the so called "hopping matrix", a part of the Dirac operator in the quasizero-mode sector. It has been done for $S U(2)$ color group and the number of fermions flavors $N_{f}=1,2,4$. Except in the last case, chiral symmetry breaking has been clearly observed, for dense enough dyon ensemble.

Larsen and myself [23] use direct numerical simulation of the instanton-dyon ensemble, both in the high-T dilute and low-T dense regime. Unlike the previous work, it uses classical dyon-antidyon interaction determined in Ref.[22]. The holonomy potential as a function of all parameters of the model is determined and minimized. In Fig. 3 from this work we show the dependence of the total free energy on holonomy value, for different ensemble densities. As one can see, at high density of the dyons their back reaction shifts the minimum to $v=1 / 2$, which is the confining value for $\mathrm{SU}(2)$ $(\cos (\pi v)=0)$ : the confinement transition is thus generated. The self-consistent parameters of the ensemble, minimizing the free energy, is determined for each density.

The next work of Larsen and myself [24] addressed the issue of chiral symmetry breaking in the $N_{c}=2$ theory with two light quark flavors $N_{f}=2$. Numerical simulations are done for partition

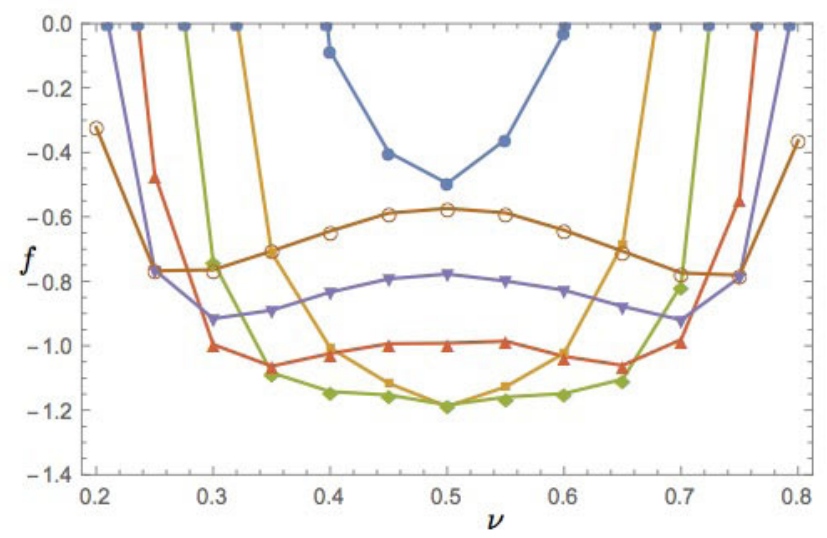

Figure 3. (Color online). Free Energy density $f$ as a function of holonomy $v$ at $S=6, M_{D}=2$ and $N_{M}=N_{L}=$ 16. The different curves corresponds to different densities. $\bullet n=0.53, \boldsymbol{\square} n=0.37, \bullet n=0.27, \boldsymbol{\Delta} n=0.20$, $\mathbf{\nabla}$ $n=0.15, \circ n=0.12$. Not all densities are shown. 
function appended by the fermionic determinant, evaluated in the zero mode approximation. Using two sizes of the system, with 64 and 128 dyons, we identify the finite-size effects in the eigenvalue distribution, and extrapolate to infinite size system. The location of the chiral transition temperature is defined both by extrapolation of the quark condensate, from below, and the so called "gaps" in the Dirac spectra, from above. We do indeed observe, for SU(2) gauge theory with 2 flavors of light fundamental quarks, that the deconfinement and chiral symmetry restoration transitions occur about at the same dyon density, see Fig.4. Determination of the precise transition points is difficult since both transitions appear to be in this case just a smooth crossovers. Those should correspond to inflection points (change of curvature) on the plots to be shown. Looking from this perspective at Fig. 4, one would locate the inflection points of both curves, for $\langle P\rangle$ or $\langle\bar{\psi} \psi\rangle$, at the same location, namely $S=7-7.5$.

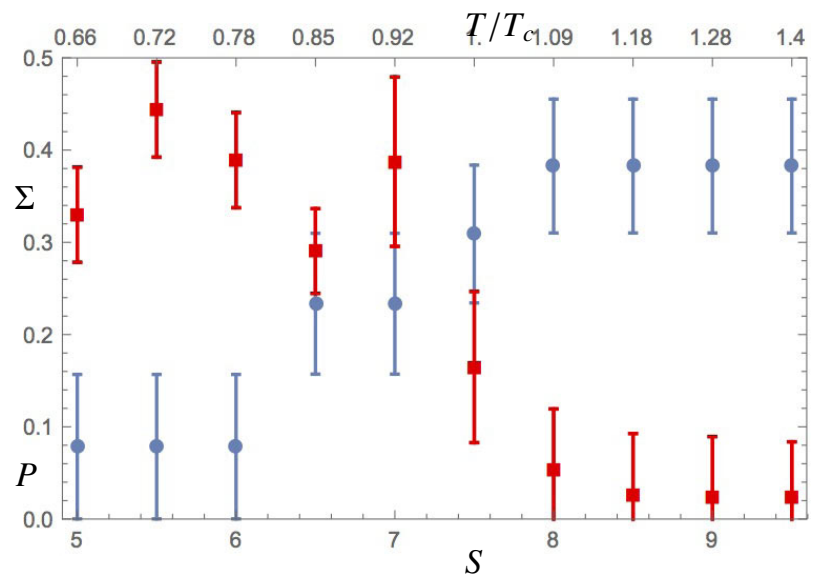

Figure 4. (Color online) The Polyakov loop $P$ (blue circles) and the chiral condensate $\Sigma$ (red squares) as a function of action $S=8 \pi^{2} / g^{2}$ or temperature $T / T_{c} . \Sigma$ is scaled by 0.2 .

\section{Quarks with nontrivial periodicity and $Z\left(N_{c}\right)$ QCD}

Periodicity condition along the Matsubara circle can be defined with some arbitrary angles $\psi_{f}$ for quarks with the flavor $f$. As was determined by van Baal and collaborators, fermionic zero mode "hops" from one type of dyon to the next at certain critical values. The resulting rule is: it belogs to the dyon corresponding to the segment of the holonomy circle $v_{i}$ to which the periodicity phase belongs: $\mu_{i}<\psi_{f}<\mu_{i+1}$.

In physical QCD all quarks are fermions, and therefore $\psi_{f}=\pi$ for all $f$. This case is schematically shown by blue dots in Fig.5(left): all fermions fall on the same segment of the circle, and therefore only one, of $N_{c}$ dyons, have zero modes and interact with quarks.

But one can introduce other arrangements of these phases. In particular, for $N_{c}=N_{f}$ the opposite extreme is the so called $Z\left(N_{c}\right)$ QCD, proposed in [30-34], put them symmetrically around the circle, see Fig.5(right). In this case, the instanton-dyon framework becomes very symmetric: each dyon interact with "its own" quark flavor.

The $Z\left(N_{c}\right)$ QCD has been studied in the mean field framework [20], by statistical simulations [25] and also by lattice simulations [35]. The first two papers consider the $N_{c}=N_{f}=2$ version of the 


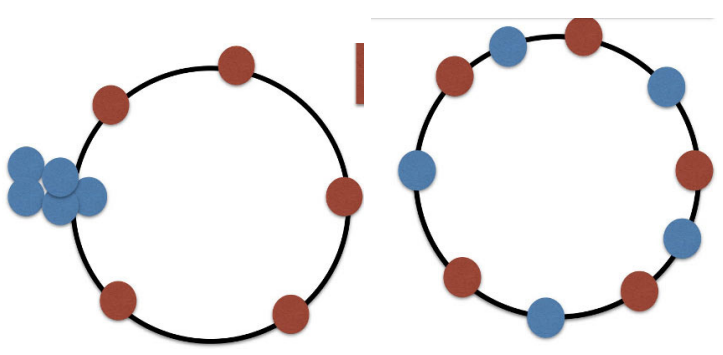

Figure 5. Schematic explanation of the difference between the usual QCD (left) and the $Z\left(N_{c}\right)$ QCD (right).

theory, while the last one focus on the $N_{c}=N_{f}=3$. In the former case the set of phases are $\psi_{f}=0, \pi$, so one quark is a boson and one is a fermion. In the latter $\psi_{f}=\pi / 3, \pi,-\pi / 3$.

All these works find deconfinement transition to strengthen significantly, compared to QCD with the same $N_{c}, N_{f}$ in which it is a very snooth crossover. While in [20] the $<P>$ reaches zero smoothly, a la second order transition, the simulations [25] and lattice [35] both see clear jump in its value indicated strong first order transition. The red squares at Fig.6(left) from [25] are comparing the behavior of the mean Polyakov line in $Z_{2}$ and ordinary QCD. The parameter $S$ used as measure of the dyon density is the "instanton action", related with the temperature by

$$
S=\left(\frac{11 N_{c}}{3}-\frac{2 N_{f}}{3}\right) \log \left(\frac{T}{\Lambda}\right)
$$

The dyons share it as $S_{M}=v S, S_{L}=\bar{v} S$. So, larger $S$ at the r.h.s. of the figure correpond to high $T$ and thus to more dilute ensemble, since densities contain $\exp \left(-S_{i}\right)$.

All three studies see a non-zero chiral condensates in the studied region of densities: perhaps no chiral restoration happens at all. The value fo the condensate are shown in Fig.6(right) from [25].

The simulation [20] demonstrate that the spectrum of the Dirac eigenvalues has a very specific "triangular" shape, characteristic of a single-flavor QCD. This explains why the $Z\left(N_{c}\right)$ QCD has much larger condensate than ordinary QCD, at the same dyon density, and also why there is no tenedecy to restoration. As expected, all works see different condensates, $<\bar{u} u>\neq<\bar{d} d>$, but with difference smaller than one could expect from the difference in the dyon density.

\section{Summary}

Studies of semiclassical theory and gauge topology shifted from instantons in the QCD vacuum to the finite temperature phenomena, and more specifically, to the mechanisms of the deconfinement and chiral restoration transitions. Incorporation of nonzero VEV of the Polyakov line - called holonomy lead to a shift from instantons to their constituents - instanton-dyons. Recent papers on the ensembles of those, done both in the mean field approximation and by direct statistical simulations, has lead to very significant advances. Unlike instantons, these objects have three different set of charges, therefore affecting holonomy value and thus allowing to tie together deconfinement and chiral restoration.

Like the instantons, dyons have topological charges and are subject to topological index theorems. It means that for appropriate fermionic bondary conditions they must have fermionic zero modes. Collectivization of those into a quark condensate follows, provided the ensemble is dense enough. Unlike instantons, the dyons posess (Euclidean) electric charges, and interact directly with the holonomy. Therefore, they back-react and are able to modify the holonomy potential. Furthrmore, as the 

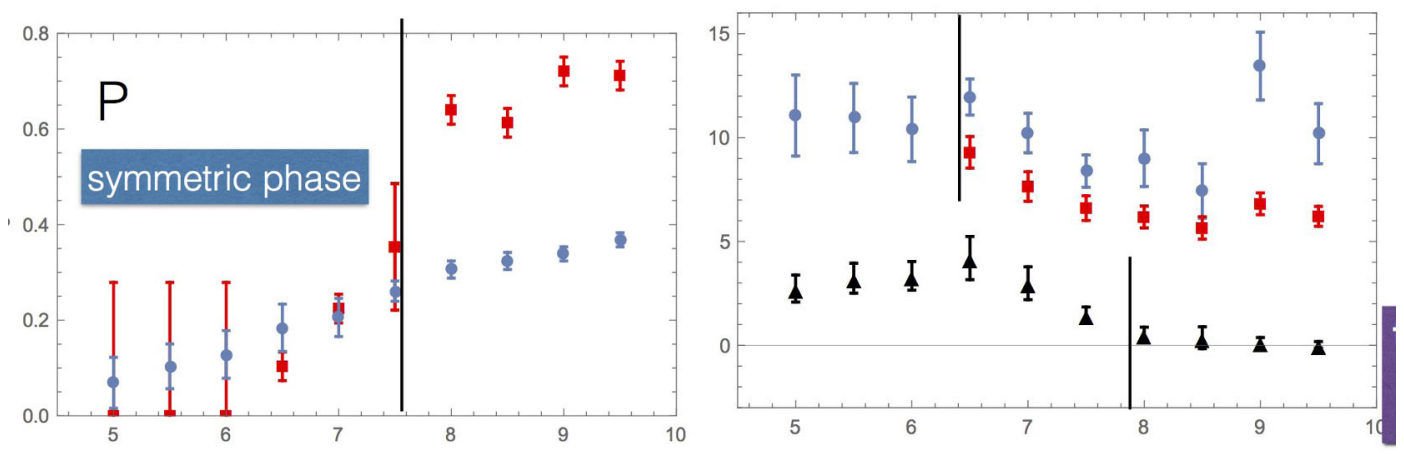

Figure 6. (left) The mean Polyakov line $\mathrm{P}$ versus the density parameter $S$. Red squares are for $Z_{2} Q C D$ while blue circles are for the usual QCD, both with $N_{c}=N_{f}=2$. (right) The quark condensate versus the density parameter $S$. Black triangles correspond to the usual QCD: and they display chiral symmetry restoration. Blue and red poins are for two flavor condensates of the $Z_{2} Q C D$ : to the left of vertical line there is a "symmetric phase" in which both types of dyons and condensates are the same.

calculations showed, the potential's minimum shifts to confining value of the holonomy, at which all types of dyons become equal.

Unlike instantons, the dyons posess magnetic charges, and thus their ensemble generates the magnetic screening mass. Recall that perturbative polarization tensor does not generate it [28]: but, according to lattice data, in the near- $T_{c}$ region it even surpasses the electric mass. While we have not discuss it above, let me just mention that it clearly indicates a transition from electric (QGP) to magnetic plasma, as the coupling grows with decreasing temperature.

This round of studies had shown why QCD with light quarks, unlike pure gauge theories, have rather smooth cross-over transition: the reason is the symmetry between different dyon kinds is broken by quarks in a very robust way: thus the "symmetric phase" - in which all dyons are represented equally - is never realized. The densities of $L, M$ dyons are always different by at least factor two or so.

It is a remarkable finding that, with the same number of light quarks but modified periodicity phases $\psi_{f}$, one gets back a strong first order deconfinement transition. Further studies with variable phase should reveal an "internal phase transitions" related with "hopping" of quark zero mode from one type of dyon to the next. Since this phenomenon has no other known explanation, apart of instanton-dyon theory, documenting those on the lattice would be crucial to finalize the mechanism of the deconfinement and chiral transitions.

My final point is an obvious one: multiple predictions of the instanton-dyon model needs to be extensively checked on the lattice. While the periodicity phases mentioned above open new windows, needless to say, those should also be supplemeneted by a systematic "hunt" for instanton-dyons on the lattice, to quantify their density and parameters directly.

\section{Acknowledgements}

The progress reported would not be possible without contributions by Pierre van Baal and Mitya Diakonov, the consequences of whose legacy remains to be worked out. The particular results reported here were obtained with my collaborators on the instanton-dyon projects, P.Faccioli, T.Sulejmanpasic, R.Larsen, I.Zahed, and Y. Liu. 


\section{References}

[1] Y. Nambu, Phys. Rev. D 10, 4262 (1974); S. Mandelstam, Phys.Rep. . 23C, 145 (1976); G. 't Hooft, in Proceedings of the European Physics Society 1975, edited by A. Zichichi (Editrice Compositori, Bologna, 1976), p.1225.

[2] Nambu Y.,Phys.Rev.Lett.4(1960)380,Phys.Rev. 117 (1960) 648, Nambu Y. and G.JonaLasinio.Phys.Rev. 122(1961)345

[3] A. D'Alessandro, M. D'Elia and E. V. Shuryak, "Thermal Monopole Condensation and Confinement in finite temperature Yang-Mills Theories," Phys. Rev. D 81, 094501 (2010) doi:10.1103/PhysRevD.81.094501 [arXiv:1002.4161 [hep-lat]].

[4] E. V. Shuryak, "The Role of Instantons in Quantum Chromodynamics. 1. Physical Vacuum," Nucl. Phys. B 203, 93 (1982).

[5] T. Schafer and E. V. Shuryak, "Instantons in QCD," Rev. Mod. Phys. 70, 323 (1998) [arXiv:hep$\mathrm{ph} / 9610451]$.

[6] T. C. Kraan and P. van Baal, "Monopole constituents inside SU(n) calorons," Phys. Lett. B 435, 389 (1998) [arXiv:hep-th/9806034].

[7] K. -M. Lee and C. -h. Lu, "SU(2) calorons and magnetic monopoles," Phys. Rev. D 58, 025011 (1998) [hep-th/9802108].

[8] D. Diakonov, Topology and Confinement, arxiv:0906.2456v1 (2009)

[9] D. Diakonov, N. Gromov, V. Petrov and S. Slizovskiy, "Quantum weights of dyons and of instantons with nontrivial holonomy," Phys. Rev. D 70, 036003 (2004) [arXiv:hep-th/0404042].

[10] N. M. Davies, T. J. Hollowood, V. V. Khoze and M. P. Mattis, Nucl. Phys. B 559, 123 (1999) doi:10.1016/S0550-3213(99)00434-4 [hep-th/9905015].

[11] E. Poppitz, T. Schaefer and M. Unsal, "Continuity, Deconfinement, and (Super) Yang-Mills Theory," JHEP 1210, 115 (2012) [arXiv:1205.0290 [hep-th]].

[12] E. Poppitz and M. Unsal, "Seiberg-Witten and 'Polyakov-like' magnetic bion confinements are continuously connected," JHEP 1107, 082 (2011) [arXiv:1105.3969 [hep-th]].

[13] D. J. Gross, R. D. Pisarski and L. G. Yaffe, “QCD and Instantons at Finite Temperature," Rev. Mod. Phys. 53, 43 (1981).

[14] E. Shuryak and T. Sulejmanpasic, "Holonomy potential and confinement from a simple model of the gauge topology," Phys. Lett. B 726, 257 (2013) [arXiv:1305.0796 [hep-ph]].

[15] R. D. Pisarski, "Towards a theory of the semi-Quark Gluon Plasma," Nucl. Phys. Proc. Suppl. 195, 157 (2009).

[16] Y. Liu, E. Shuryak and I. Zahed, “Confining dyon-antidyon Coulomb liquid model. I.,” Phys. Rev. D 92, no. 8, 085006 (2015) [arXiv:1503.03058 [hep-ph]].

[17] Y. Liu, E. Shuryak and I. Zahed, "Light quarks in the screened dyon-antidyon Coulomb liquid model. II.,” Phys. Rev. D 92, no. 8, 085007 (2015) [arXiv:1503.09148 [hep-ph]].

[18] Y. Liu, E. Shuryak and I. Zahed, “The Instanton-Dyon Liquid Model III: Finite Chemical Potential," arXiv:1606.07009 [hep-ph].

[19] Y. Liu, E. Shuryak and I. Zahed, "Light Adjoint Quarks in the Instanton-Dyon Liquid Model IV," arXiv:1605.07584 [hep-ph].

[20] Y. Liu, E. Shuryak and I. Zahed, "The Instanton-Dyon Liquid Model V: Twisted Light Quarks," arXiv:1606.02996 [hep-ph].

[21] P. Faccioli and E. Shuryak, "QCD topology at finite temperature: Statistical mechanics of selfdual dyons,” Phys. Rev. D 87, no. 7, 074009 (2013) [arXiv:1301.2523 [hep-ph]]. 
[22] R. Larsen and E. Shuryak, "Classical interactions of the instanton-dyons with antidyons," Nucl. Phys. A 950, 110 (2016) [arXiv:1408.6563 [hep-ph]].

[23] R. Larsen and E. Shuryak, "Interacting ensemble of the instanton-dyons and the deconfinement phase transition in the SU(2) gauge theory," Phys. Rev. D 92, no. 9, 094022 (2015) [arXiv:1504.03341 [hep-ph]].

[24] R. Larsen and E. Shuryak, "Instanton-dyon Ensemble with two Dynamical Quarks: the Chiral Symmetry Breaking,” Phys. Rev. D 93, no. 5, 054029 (2016) [arXiv:1511.02237 [hep-ph]].

[25] R. Larsen and E. Shuryak, "Instanton-dyon Ensembles III: Exotic Quark Flavors," arXiv:1605.07474 [hep-ph].

[26] V. G. Bornyakov, E.-M. Ilgenfritz, B. V. Martemyanov and M. Mueller-Preussker, Phys. Rev. D 93, no. 7, 074508 (2016) doi:10.1103/PhysRevD.93.074508 [arXiv:1512.03217 [hep-lat]].

[27] A. Bazavov and P. Petreczky, "Polyakov loop in 2+1 flavor QCD," Phys. Rev. D 87, no. 9, 094505 (2013) [arXiv:1301.3943 [hep-lat]].

[28] E. V. Shuryak, Sov. Phys. JETP 47, 212 (1978) [Zh. Eksp. Teor. Fiz. 74, 408 (1978)].

[29] F. Karsch and M. Lutgemeier, "Deconfinement and chiral symmetry restoration in an SU(3) gauge theory with adjoint fermions," Nucl. Phys. B 550, 449 (1999) [hep-lat/9812023].

[30] H. Kouno, Y. Sakai, T. Makiyama, K. Tokunaga, T. Sasaki and M. Yahiro, J. Phys. G 39, 085010 (2012).

[31] Y. Sakai, H. Kouno, T. Sasaki and M. Yahiro, Phys. Lett. B 718, 130 (2012) [arXiv:1204.0228 [hep-ph]].

[32] H. Kouno, T. Makiyama, T. Sasaki, Y. Sakai and M. Yahiro, J. Phys. G 40, 095003 (2013) [arXiv:1301.4013 [hep-ph]].

[33] H. Kouno, T. Misumi, K. Kashiwa, T. Makiyama, T. Sasaki and M. Yahiro, Phys. Rev. D 88, no. 1, 016002 (2013) [arXiv:1304.3274 [hep-ph]].

[34] H. Kouno, K. Kashiwa, J. Takahashi, T. Misumi and M. Yahiro, arXiv:1504.07585 [hep-ph].

[35] T. Misumi, T. Iritani and E. Itou, "Finite-temperature phase transition of $N_{f}=3$ QCD with exact center symmetry," arXiv:1510.07227 [hep-lat]. 